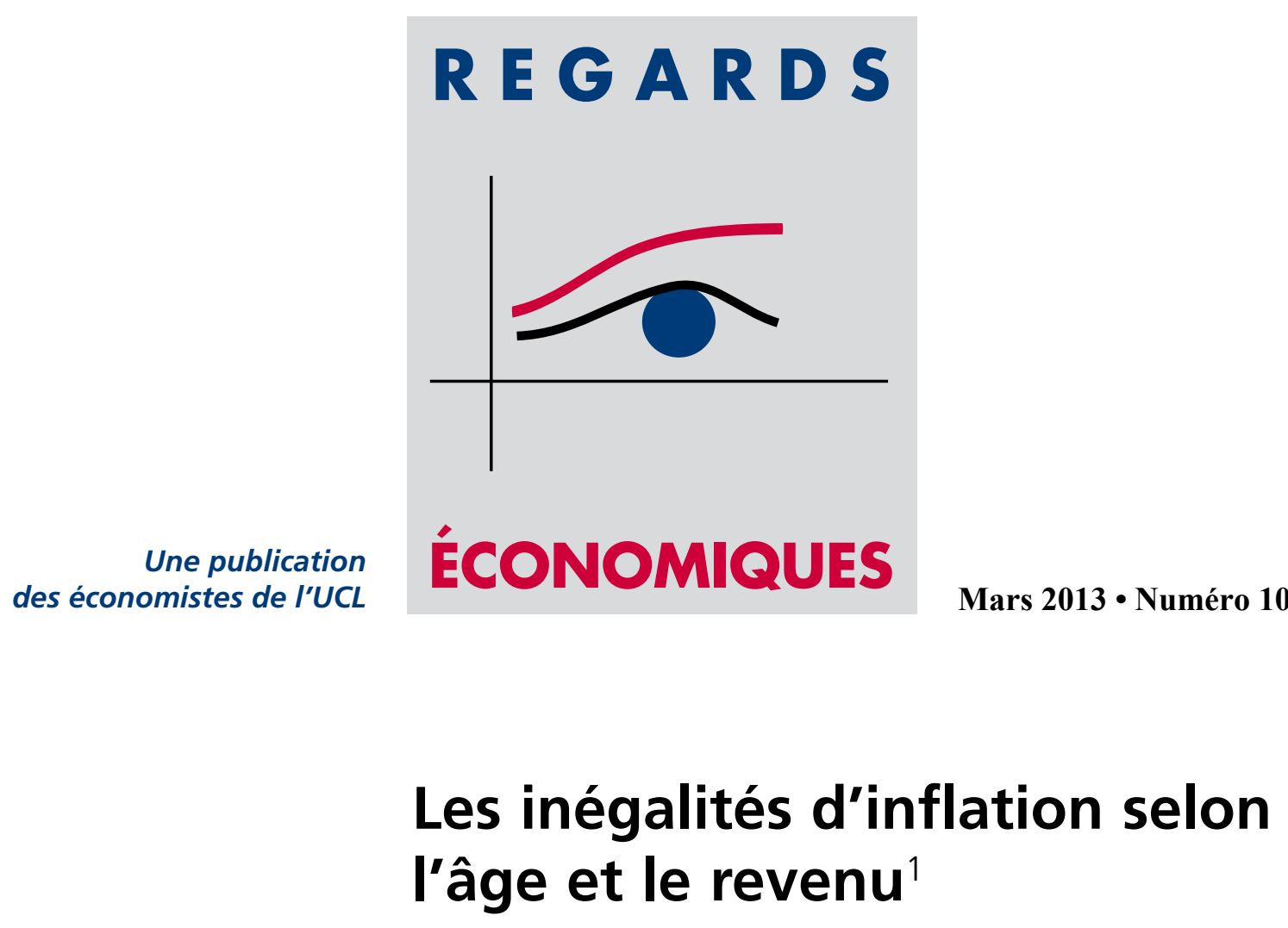

Cette étude montre qu'il existe en Belgique une inégalité d'inflation entre les ménages de niveaux de revenu et d'âges différents. Sur la période 20012011, l'inflation cumulée est proche de $32 \%$ pour le décile le plus pauvre contre $26 \%$ pour le décile le plus riche. L'inflation cumulée atteint également $32 \%$ pour les 70 ans et plus contre $26 \%$ pour les 25-29 ans. Sur la même période, l'inflation qui sert de référence pour l'indexation est de $24 \%$, ce qui signifie une perte de pouvoir d'achat potentielle de $9 \%$ en dix ans pour les ménages les plus pauvres et les $\geq 70$ ans. Notre système d'indexation protège donc moins bien les ménages les plus vulnérables. Notre étude montre également que les inégalités d'inflation ont augmenté sur la période, principalement en raison de la forte hausse des prix de l'énergie entre 2004 et 2008. Un mécanisme de protection comme les chèques mazout semble donc tout à fait justifié.

\section{Vincent Bodart}

Jean Hindriks veau général des prix des biens et des services consommés par les ménages sur le territoire national. C'est une mesure synthétique des évolutions de prix. L'IPC a un rôle socio-économique important : l'IPC, publié par le SPF Economie, sert à indexer les contrats privés, les salaires dans le secteur public et dans le secteur privé, les pensions alimentaires, les rentes viagères, de même que les allocations sociales, le revenu d'intégration et les pensions. L'indice retenu pour les besoins de l'indexation est souvent l'indice santé qui est l'IPC hors alcool, tabac et tous les carburants.

L'IPC est un indice synthétique dit «de Laspeyres» basé sur une année de référence. Outre la composition de l'échantillon de produits, les pondérations utilisées pour agréger les groupes de produits sont calculées pour une même année de référence. Ces pondérations représentent la part des dépenses associées au groupe de produits au sein de l'ensemble des dépenses de consommation des ménages couvertes par 1'IPC. En Belgique, ces pondérations ne sont révisées en moyenne que tous les 8 ans. Les poids actuels sont ceux de 2004 et les adaptations de l'échantillon de produits en fonction de nouveaux biens ou services sont très rares (voir infra). ${ }^{2}$

\footnotetext{
${ }^{1}$ Nous remercions Muriel Dejemeppe ainsi que deux lecteurs anonymes pour leur relecture attentive du texte et pour leurs commentaires et remarques. Nous remercions aussi vivement Monsieur Vincent Coutton, de la Direction Générale de la Statistique et de l'Information Economique du SPF Economie, pour nous avoir fourni les données de l'enquête sur le budget des ménages et différentes explications utiles.

${ }^{2} \mathrm{Il}$ existe dans les faits un second indice des prix en Belgique, l'Indice harmonisé (IPCH) pour lequel les adaptations de l'échantillon aux nouveaux produits sont plus fréquentes, et pour lequel les pondérations des différentes catégories de produits peuvent être révisées chaque année sur base des dépenses de consommation des ménages. Ce n'est cependant pas cet indice qui est utilisé pour calculer l'indice-santé qui sert de référence à l'indexation des salaires et allocations. Nous ne ferons donc pas référence à cet indice par la suite.
} 
Comme l'indice des prix à la consommation mesure l'évolution des prix d'un ensemble de biens et services représentant la consommation de l'ensemble des ménages, les poids utilisés correspondent à la structure de consommation moyenne. Mais le consommateur «moyen» n'existe pas. Chaque ménage a sa propre structure de consommation, qui diffère plus ou moins fortement de la structure générale : le loyer absorbe une part plus importante du budget des ménages locataires, les dépenses de santé pèsent en général plus lourd dans celui des personnes âgées et les dépenses de tabac dépendent de la présence de fumeurs dans le ménage. La hausse des prix à laquelle vous êtes personnellement confronté dépend donc de ce que vous achetez : si vous consommez beaucoup d'un produit dont le prix augmente plus que la moyenne, votre «inflation individuelle» sera plus élevée que l'inflation moyenne.

Le coût de la vie n'évolue donc pas forcément de la même manière pour tous. Ainsi, dans la mesure où la composition des achats de biens et services effectués par chaque ménage peut différer du panier représentatif utilisé pour le calcul de l'indice général des prix à la consommation, il y a tout lieu de penser que le taux d'inflation calculé pour un ménage particulier ou une catégorie particulière de ménages pourra s'écarter de façon plus ou moins marquée du taux d'inflation moyen. Cela est d'autant plus vrai que les prix de certains biens augmentent plus que d'autres. Les études existantes sur le sujet, et qui portent sur des pays étrangers, établissent clairement de telles différences. L'étude menée par Hobijn et Lagakos (2003) pour les Etats-Unis fait par exemple état de différences significatives entre le taux d'inflation supporté par les ménages âgés (plus de 61 ans) et celui supporté par les ménages des autres catégories d'âge.

Dans ce numéro de Regards Economiques, nous avons cherché à mesurer les écarts d'inflation qui existent selon les niveaux de revenu et selon les catégories d'âge. Il y a au moins deux raisons pour lesquelles l'indice général (ou «moyen») des prix à la consommation pourrait ne pas refléter correctement l'évolution du coût de la vie de groupes de ménages de revenus différents ou de classes d'âge différentes. ${ }^{3}$

La première raison est le caractère «plutocratique» de l'indice général, dans la mesure où cet indice «moyen» reflète une structure de consommation moyenne de l'ensemble des ménages, pondérée par l'importance relative de ces ménages dans la dépense totale (voir l'annexe 1 dans Regards économiques n46). Cela signifie que les ménages à revenu élevé ont un poids plus important dans le calcul de l'indice «moyen» du coût de la vie que les ménages à faible revenu. Comme la structure de consommation change fortement avec le niveau de revenu (cf. infra), la sous-représentation des ménages à faible revenu pourrait créer un écart important entre leur évolution effective du coût de la vie et celle reflétée par l'indice «moyen» du coût de la vie. Cet argument s'applique aussi aux différentes classes d'âge dans la mesure où les revenus varient selon l'âge en commençant par augmenter en début de carrière pour ensuite diminuer avec le départ à la pension.

La deuxième raison est liée à l'évolution de la structure de consommation au cours du temps. En Belgique comme dans d'autres pays, l'indice «moyen» du coût de la vie est basé sur une structure de consommation moyenne établie pour une année de référence. Cela a pour effet de ne pas prendre en considération les évolutions de la structure de consommation au fil du temps avec les possibilités de substitutions entre biens que cela implique. En particulier, deux formes de substitution

\footnotetext{
${ }^{3}$ Il y a une autre raison que nous ne traitons pas (faute de données suffisantes) qui est que les prix pour un même bien ou service peuvent différer selon la catégorie de ménage. C'est le cas pour les tarifs sociaux en matière de gaz et électricité, des soins médicaux VIPO, des logements sociaux. C'est aussi le cas des produits pour lesquels les fournisseurs adoptent un certain degré de différentiation horizontale (variétés différentes) ou verticale (différents niveaux de qualité). Cette différentiation des prix n'est pas prise en compte dans l'analyse.
} 
sont possibles : la substitution «choisie» et la substitution «forcée». La première permet d'atténuer l'effet des variations de prix sur le niveau de vie alors que la seconde accentue ces effets. Les indices du coût de la vie utilisés habituellement ignorent ces effets de substitution. Cela cache une différence importante possible entre riches et pauvres. Les premiers pourraient en effet opérer des substitutions «choisies» des biens devenus plus chers vers les biens devenus moins chers, avec pour effet d'atténuer l'effet de l'inflation sur leur coût de la vie. En revanche, les pauvres pourraient être sujets à des substitutions «forcées» vers des biens devenus plus chers et dont la consommation est difficilement ajustable à la baisse (comme par exemple, le mazout de chauffage). Il en va de même pour les dépenses de santé des personnes âgées qui peuvent difficilement ajuster leur consommation en fonction de la hausse du prix des médicaments. Pour plus de précisions sur les effets de substitution et l'inflation, nous renvoyons le lecteur à Regards économiques nº45.

Dans notre étude de 2006 publiée dans Regards économiques n²6, nous avions calculé le taux d'inflation effectif qui s'applique aux personnes de revenus différents en tenant compte explicitement de la différence des structures de consommation et de son évolution au cours du temps au travers des enquêtes récurrentes sur le budget des ménages qui sont menées par la Direction générale de la Statistique et de l'Information Economique (DGSIE) du SPF Economie. De la sorte, nous avions intégré progressivement et en partie les substitutions effectuées par les consommateurs, à la place de l'actuel panier fixe. Notre analyse de 2006, portant sur la période 1998-2005, avait révélé que les ménages à revenus faibles ont une structure de consommation sensiblement différente des ménages à revenus élevés et que la structure de consommation évolue au fil du temps. Malgré ces différences, nous arrivions au résultat assez surprenant d'une faible différence d'inflation entre les ménages à faible revenu et les ménages à revenu élevé. Par exemple, le taux d'inflation générale supporté par les ménages du décile 1 (les plus pauvres) entre 1998 et 2005 était de 14,6\%, tandis que celui supporté par les ménages du décile 10 (les plus riches) était de 13,8\%. Même durant les années marquées par une forte hausse des prix du pétrole, comme en 2005 par exemple, la différence de taux d'inflation restait faible entre les différentes catégories de ménages, alors qu'on aurait pu s'attendre à un élargissement de l'écart étant donné le poids nettement plus important des dépenses d'habitation pour les ménages à bas revenu par rapport aux ménages à haut revenu. Ainsi, en 2005, le taux d'inflation générale calculé pour les ménages du décile 1 était de $2,6 \%$, alors que celui calculé pour les ménages du décile 10 était de 2,3\%. La conclusion de notre analyse était donc que l'indice général des prix à la consommation national était une assez bonne approximation du taux d'inflation effectif supporté par des ménages de niveaux de revenu différents.

Dans ce numéro de Regards économiques, nous actualisons notre analyse de 2006 en examinant les écarts d'inflation sur la période 2000-2011. Nous allons aussi aborder la question de savoir si l'inflation varie selon les catégories d'âge, question qui n'était pas traitée dans notre étude de 2006.

La suite de l'article est organisée de la façon suivante. Nous commencerons par présenter la méthodologie que nous avons suivie pour calculer l'inflation supportée par des ménages de niveaux de revenu et d'âges différents. Nous présenterons ensuite nos résultats. Les implications de notre étude, de même que certaines limites, seront discutées en conclusion.

\section{Méthodologie}

Dans notre étude, l'évolution du coût de la vie pour un ménage particulier est mesurée par l'indice de prix de Laspeyres. En cela, notre approche est similaire à celle qui est utilisée en Belgique par le Service de l'Indice pour le calcul de l'indice officiel des prix à la consommation national et par la plupart des instituts de statistiques dans le monde. Nous calculons ensuite l'inflation supportée par un 
... Méthodologie ménage particulier entre deux périodes adjacentes (par exemple entre les années $\mathrm{t}-1$ et $\mathrm{t}$ ) comme une moyenne pondérée du taux de croissance de l'indice du prix des différents produits qui composent le panier de biens et services achetés par ce ménage. Dans le calcul de la moyenne, la pondération attribuée au prix de chaque produit correspond à la part de la dépense totale du ménage qui est consacrée à l'achat de ce produit. Dans le calcul que nous effectuons, les pondérations varient de période à période, de manière à rendre compte au mieux de la structure des dépenses des ménages à chaque période (c'est-à-dire tenir compte des effets de substitution). A ce niveau, notre méthode de calcul s'écarte de celle qui est utilisée par le Service de l'Indice, qui utilise des pondérations fixes pour le calcul des indices officiels des prix à la consommation. ${ }^{4}$

Sous une forme algébrique, notre méthode de calcul de l'inflation est donnée par l'expression suivante :

$$
\text { (1) } \Pi_{t}=\sum_{j=1, \ldots, m} w_{j, t-1} \Pi_{j, t}
$$

où $\Sigma$ est l'opérateur de somme, $\Pi_{t}$ est le taux d'inflation générale calculé entre les périodes $t-1$ et $t, \Pi_{j, t}$ est le taux de croissance du prix du produit $\mathrm{j}$ entre les périodes $\mathrm{t}-1$ et $\mathrm{t}, \mathrm{w}_{\mathrm{j}, \mathrm{t}-1}$ est le coefficient de pondération associé au produit $\mathrm{j}$ et calculé à la période $\mathrm{t}-1$, et $\mathrm{m}$ indique le nombre de produits qui composent le panier de biens et services du ménage considéré.

Dans notre étude, nous calculons le taux d'inflation donné par l'expression (1) pour différents groupes de ménages, définis en fonction (a) du niveau du revenu du ménage comme cela est expliqué plus loin, et (b) de la catégorie d'âge du ménage. Dans la mesure où nous supposons que le prix payé pour un produit donné est le même pour les différentes catégories de ménages, ce n'est que si la structure des dépenses varie entre catégories de ménages que l'on relèvera une différence dans le taux d'inflation supporté par chaque catégorie de ménages. ${ }^{5}$

Pour calculer le taux d'inflation donné par l'expression (1) pour les différentes catégories de ménages, deux types de données statistiques sont nécessaires :

- Les indices de prix pour les biens et services qui sont achetés par les ménages, grâce auxquels on pourra calculer les taux d'inflation $\Pi_{\mathrm{j}, \mathrm{t}}$. Les données que nous avons utilisées sont les indices officiels de prix par produit publiés mensuellement par le SPF Economie. Elles couvrent la période allant de janvier 2000 à décembre 2011. Des moyennes annuelles ont été calculées pour chaque indice de prix.

- Les montants dépensés par les ménages pour chaque produit, ces données étant nécessaires pour calculer les pondérations $\mathrm{w}_{\mathrm{j}, \mathrm{t}-1}$. Ces données sont reprises de l'enquête sur le budget des ménages (EBM) réalisée en Belgique par la DGSIE du SPF Economie (pour plus de détails sur cette enquête, voir l'encadré 1 dans Regards économiques $\left.\mathrm{n}^{\circ} 46\right){ }^{6}$ Pour notre étude, les données de l'EBM ont été réparties en 10 déciles en prenant comme critère de répartition le niveau de revenu par ménage. ${ }^{7}$ Dans la suite de l'analyse, le décile 1 correspond à la catégorie de

\footnotetext{
$\overline{{ }^{4} \text { Les pondérations fixes qui sont utilisées par le Service de l'Indice sont déterminées à partir de la structure des dépenses de consom- }}$ mation relevées au cours d'une période donnée, qui constitue la période de base de l'indice. Pour une présentation de la méthode de calcul utilisée par le Service de l'Indice, voir Ministère des affaires économiques (1999).

${ }^{5}$ A notre connaissance, il n'existe pas en Belgique de statistiques officielles de prix pour des catégories différentes de ménages. Nous ne disposons pas non plus d'information sur l'importance de la consommation des produits blancs ou des tarifs sociaux selon les catégories de ménage. C'est une lacune qui pourrait être prochainement levée par l'utilisation des données de scanning en magasin. ${ }^{6}$ Ces enquêtes présentent quelques limites. La taille de l'échantillon est très faible (moins de 4000 ménages à répartir en dix classes de revenu), la définition des revenus (hors transferts privés) est basée sur les déclarations et ne représentent qu'une partie des moyens disponibles pour les ménages, les déclarations ne concernent qu'un mois de l'année avec possibilité de variation des dépenses et des revenus d'un mois à l'autre.

${ }^{7}$ Dans notre cas, un décile est chacune des 9 valeurs de revenu qui divisent l'échantillon de ménages en 10 parts égales, de sorte que chaque partie représente 1/10 de l'échantillon.
} 
... Méthodologie revenu la plus faible, tandis que le décile 10 correspond à la catégorie de revenu la plus élevée. Nous adoptons aussi un autre critère de répartition qui est la classe d'âge et, à ce effet, nous utilisons les données de l'EBM réparties en 11 catégories d'âge différentes (<25 ans, 25-29, 30-34, 35-39, 40-44, 45-49, 50-54, 55-59, 60$64,65-69, \geq 70$ ans). Les données de l'EBM dont nous avons pu disposer portent sur la période $1999-2010{ }^{8}$

Pour calculer l'inflation, il ne s'agit pas seulement de suivre les prix et leur évolution, mais aussi les tendances en matière de consommation (voir l'encadré 1). ${ }^{9} \mathrm{La}$ liste des articles qui composent l'indice (et il y en a actuellement 520 répartis en 12 catégories) doit donc être revue et corrigée. Mais pas n'importe quand, ni comment. C'est là un des rôles de la Commission de l'Indice. Ces révisions concernent les articles, leur pondération et l'échantillon de magasins pour l'enregistrement des prix. Elles sont basées sur une enquête générale sur les dépenses des ménages belges. Raison pour laquelle elles ne sont pas fréquentes : il n'y a ainsi eu que huit révisions depuis l'instauration de l'indice en 1920, dont cinq ces quarante dernières années - en 1974-1975, 1981, 1988, 1996 et 2004 -, preuve que les tendances en matière de consommation évoluent de plus en plus vite. Mais un laps de temps de huit ans en moyenne entre deux révisions, n'est-ce pas encore de trop ? Risquant de rendre l'indice moins représentatif, voire démodé ? Afin de pallier cet inconvénient, la Commission de l'Indice a décidé, lors de la réforme de 2004, d'effectuer une actualisation bisannuelle. Il y en a eu une en 2008 et une deuxième en 2010. Depuis lors, la Commission prépare la grande révision qui entrera en vigueur en 2014. L'idée est que l'indice se rapproche encore plus de la réalité. Pour cela, il convient de partir non pas des relevés de prix des articles mais des données scannées, système déjà utilisé aux Pays-Bas, en Suède, Norvège et Suisse, et dont la faisabilité est actuellement étudiée par la DG Statistique belge. De quoi tenir compte non seulement des quantités achetées, mais aussi des promotions $(2+1$ gratuit, par exemple) et autres soldes. Par ailleurs, la représentativité des produits blancs et des marques de distributeurs s'en trouvera améliorée. Des marques qui sont moins chères que les marques nationales et de plus en plus proposées aux clients (en lieu et place des marques nationales) et donc consommées. ${ }^{10}$

De manière à pouvoir mettre correctement en évidence les différences possibles d'inflation entre ménages, il est recommandé de prendre les données de prix et de dépenses par produit au niveau de désagrégation le plus bas. ${ }^{11}$ Ceci doit toutefois être effectué en veillant à obtenir la meilleure correspondance possible entre les produits (ou groupes de produits) pris en considération dans les deux bases de données. Compte tenu de cette contrainte, les données que nous avons utilisées concernent 78 produits ou groupes de produits différents. Le détail de cette classification est donné dans l'annexe $1 \mathrm{du}$ Regards économiques $\mathrm{n}^{\circ} 46$.

Il est important de souligner que les résultats des enquêtes sur les budgets des ménages sont les seules données que nous avons utilisées pour calculer les coefficients de pondération attribués à chacun des 78 groupes de produits, alors que les pondérations qu'utilise le Service de l'Indice pour calculer l'indice général des prix à la consommation sont établies en prenant en considération d'autres sources

\footnotetext{
${ }^{8}$ L'enquête 2010 est la dernière enquête pour laquelle les résultats sont disponibles.

${ }^{9} \mathrm{Si}$ la consommation baisse en volume dans la même proportion que la hausse du prix d'un produit, le montant dépensé pour ce produit est constant de même que sa pondération dans le calcul de l'inflation globale. C'est par exemple le cas si le prix des ampoules électriques double et que leur durée de vie double aussi. Les évolutions dans la qualité des produits affectent donc à la fois les prix et les quantités consommées.

${ }^{10}$ Notons que ces réformes ne concernent pas l'adaptation de l'échantillon à l'arrivée de nouveaux produits (ampoules LED) et à la disparition de certains produits (ampoules à incandescence).

${ }^{11}$ Pour la période 2000-2011, les indices de prix sont disponibles pour 520 produits différents, tandis que l'enquête sur le budget des ménages répartit les dépenses des ménages en environ 170 biens et services différents.
} 


\section{... Méthodologie}

d'information que l'EBM. ${ }^{12}$ Comme le fait le Service de l'Indice, nous avons exclu des résultats de l'enquête EBM les loyers imputés pour les logements occupés par leurs propriétaires, ces dépenses ne constituant pas de véritables dépenses de consommation. ${ }^{13}$ Nous n'avons en revanche pas appliqué les autres ajustements mineurs pratiqués par le Service de l'Indice. ${ }^{14}$ De l'explication qui précède, il résulte que les pondérations que nous avons construites ne sont pas identiques à celles calculées par le Service de l'Indice.

\section{ш Décomposition du taux d'inflation générale}

Supposons que le panier de biens qui est utilisé pour calculer l'indice général des prix à la consommation soit composé de 2 biens, le bien 1 dont les prix est $P_{1}$ et le bien 2 dont le prix est $P_{2}, P_{1}$ et $P_{2}$ étant exprimés sous forme d'indice. ${ }^{15}$

Compte tenu de la composition du panier de biens, l'indice général des prix à la consommation, $\mathrm{P}$, est construit très simplement, comme la somme pondérée de l'indice de prix du bien 1 et de l'indice de prix du bien 2 :

$$
\text { (1) } P=\alpha_{1} P_{1}+\alpha_{2} P_{2}
$$

où $\alpha_{i}(i=1,2)$ est un coefficient de pondération égal à la part des dépenses consacrées par les ménages à la consommation du bien i dans le montant total de leurs dépenses de consommation.

Dans notre exemple, comme le panier du consommateur ne comprend que 2 biens, on a bien entendu que : $\alpha_{2}=1-\alpha_{1}$.

Le taux d'inflation entre 2 périodes (par exemple t et t-1) étant défini comme le taux de croissance de l'indice général des prix à la consommation entre ces 2 périodes, il est donné par l'expression suivante :

$$
\text { (2) } \pi_{t}=\frac{P_{t}-P_{t-1}}{P_{t-1}}
$$

où $P_{t}$ est l'indice général à la période t et $P_{t-1} l^{\prime}$ indice général à la période qui précède $(\mathrm{t}-1){ }^{\mathrm{t}}{ }^{16} \mathrm{Si}$ on considère que les pondérations qui sont utilisées pour construire l'indice général des prix sont révisées à chaque période, comme c'est le cas dans notre approche, le taux d'inflation peut être réécrit de la manière suivante, en substituant (1) dans (2):

$$
\text { (3) } \pi_{t}=\frac{\left(\alpha_{1, t} P_{1, t}+\alpha_{2, t} P_{2, t}\right)-\left(\alpha_{1, t-1} P_{1, t-1}+\alpha_{2, t-1} P_{2, t-1}\right)}{P_{t-1}}
$$

Cette expression peut être réécrite de la manière suivante :

$$
\text { (4) } \pi_{t}=\frac{\left(\alpha_{1, t} P_{1, t}-\alpha_{1, t-1} P_{1, t-1}\right)}{P_{t-1}}+\frac{\left(\alpha_{2, t} P_{2, t}-\alpha_{2, t-1} P_{2, t-1}\right)}{P_{t-1}}
$$

Dans cette expression, le premier terme de droite mesure la contribution du prix du bien 1 à l'inflation générale entre t et t-1 et le second terme mesure la contribution du bien 2 à l'inflation générale. C'est de cette manière que sont calculées les contributions à l'inflation générale des différentes catégories de prix qui sont présentées dans nos différents tableaux. Ces contributions peuvent être exprimées en valeur, comme c'est le cas dans l'expression (4), ou en pourcentage du taux d'inflation générale.

\footnotetext{
${ }_{12}$ Voir Ministère des affaires économiques (1999, p.34).

${ }^{13}$ Voir Ministère des affaires économiques (1999, p.33).

${ }^{14}$ Voir Ministère des affaires économiques (1999, pp.33-34).

${ }^{15}$ Les concepts présentés dans l'encadré sont inchangés si on prend un panier composé de $\mathrm{n}$ biens au lieu de 2 biens.

${ }^{16}$ Notez que les 2 périodes ne doivent pas nécessairement être concomitantes, comme c'est par exemple le cas lorsque nous calculons l'inflation cumulée entre 2000 et 2011.
} 
En examinant l'expression (4), on peut constater que la contribution du bien i à l'inflation générale dépend de la variation du prix du bien i entre la période t-1 et la période $t$, mais aussi de la variation entre $t-1$ et $t$ de la pondération accordée au bien i dans l'indice général des prix. Cela apparaît plus clairement lorsque, par le biais de quelques manipulations simples, on réécrit l'expression (4) de la manière suivante :

$$
\text { (5) } \pi_{t}=\left[\frac{\alpha_{1, t}\left(P_{1, t}-P_{1, t-1}\right)+P_{1, t-1}\left(\alpha_{1, t}-\alpha_{1, t-1}\right)}{P_{t-1}}\right]+\left[\frac{\alpha_{2, t}\left(P_{2, t}-P_{2, t-1}\right)+P_{2, t-1}\left(\alpha_{2, t}-\alpha_{2, t-1}\right)}{P_{t-1}}\right]
$$

On voit bien, à partir de cette dernière expression, que même si le prix d'un bien ne varie pas entre 2 périodes, sa contribution à l'inflation générale sera néanmoins non nulle si le poids de ce bien dans l'indice général des prix a varié entre les 2 périodes considérées.

Rappelons enfin que l'indice officiel des prix à la consommation est établi à partir de pondérations constantes. De ce fait, l'inflation générale entre 2 périodes ne dépend que des variations de prix des différents biens qui entrent dans la composition du panier de biens. ${ }^{17}$

Le tableau 1 présente l'évolution cumulée des prix sur la période 2001-2011 obtenue en regroupant les 78 catégories de produits en 12 catégories plus importantes de dépenses (pour des raisons évidentes de présentation). On constate une évolution contrastée des prix. Le poste logement $(\mathrm{C} 4)$ a enregistré la plus forte hausse des prix avec une hausse de $+53,2 \%$, suivi du poste Horeca (C11) avec une hausse des prix de $39 \%$, du poste Alimentation (C1) avec une hausse de $33,6 \%$, du poste Assurances et autres (C12) avec une hausse de $29 \%$, du poste Transports (C7) avec une hausse des prix de $28,4 \%$, du poste Meubles et électroménager (C5) avec une hausse 19,6\% et Dépenses de santé (C6) avec une hausse de 11,7\%. A l'inverse, le poste Communication (C8) enregistre une baisse de prix de - $15 \%$. L'inflation globale moyenne est de $27,3 \%$ dont presque un tiers est attribuable au seul poste Habitation (C4) et un autre tiers est attribuable à l'inflation combinée des postes Alimentation et Transports (C1 et C7).

Tableau 1. Taux d'inflation par groupe de dépenses sur la période 2001-2011

Source : SPF Economie et calcul des auteurs

\begin{tabular}{llccc}
\hline & \multirow{2}{*}{ Taux d'inflation par grands groupes de dépenses } & \multirow{2}{*}{$\begin{array}{c}\text { Inflation } \\
\text { cumulée }\end{array}$} & \multicolumn{2}{c}{ Contribution à l'inflation globale } \\
\cline { 4 - 5 } & & 33,6 & 5,0 & 18,3 \\
\hline C1 & Alimentation et boissons non alcoolisées & 30,9 & 0,7 & 2,6 \\
\hline C2 & Alcool et tabac & 10,2 & 0,6 & 2,0 \\
\hline C3 & Articles d'habillement et chaussures & 53,2 & 7,7 & 28,2 \\
\hline C4 & Habitation (loyers, chauffage, électricité, eau) & 19,6 & 1,6 & 5,7 \\
\hline C5 & Meubles et appareils ménagers & 11,7 & 0,6 & 2,3 \\
\hline C6 & Dépenses de santé & 28,4 & 4,4 & 16,0 \\
\hline C7 & Transports & $-15,1$ & $-0,5$ & $-1,8$ \\
\hline C8 & Communication & 12,3 & 1,7 & 6,1 \\
\hline C9 & Loisirs et culture & 25,5 & 0,2 & 0,8 \\
\hline C10 & Enseignement & 39,0 & 2,2 & 8,0 \\
\hline C11 & Restaurants et hôtels & 29,0 & 3,2 & 11,8 \\
\hline C12 & Autres (assurances, soins corporels, ...) & 27,3 & 27,3 & 100,00 \\
\hline & Taux d'inflation générale & & & \\
\hline
\end{tabular}

\footnotetext{
${ }^{17}$ Les indices actuels utilisent des pondérations qui datent de 2004.
} 


\section{... Méthodologie}

Tableau 2. Structure des dépenses des ménages par déciles de niveau de revenu (en \%)

Source : SPF Economie et calcul des auteurs
Le tableau 2 présente la structure des achats de biens et services du premier décile et du dernier décile de revenu des ménages, obtenue en regroupant les 78 catégories de produits en 12 catégories de dépenses. Pour des raisons de clarté, nous avons limité la présentation des résultats aux ménages du décile de revenu 1 (les plus pauvres) et aux ménages du décile de revenu 10 (les plus riches). Nous incluons également les résultats obtenus pour l'ensemble des ménages (cf. colonne Royaume), de même que l'écart-type des pondérations pour les 10 déciles. L'écart-type des pondérations est une mesure de l'écart moyen autour de la pondération moyenne indiquée dans la colonne Royaume. La structure des dépenses de consommation est donnée pour deux périodes différentes : 2000 et 2010.

\begin{tabular}{llcccc}
\hline & 2000 & Décile 1 & Décile 10 & Royaume & Ecart-type \\
\hline C1 & Alimentation et boissons non alcoolisées & 18,7 & 13,3 & 15,6 & 1,7 \\
\hline C2 & Alcool et tabac & 3,5 & 2,5 & 2,9 & 0,4 \\
\hline C3 & Articles d'habillement et chaussures & 4,2 & 6,7 & 5,8 & 1,0 \\
\hline C4 & Habitation (loyers, chauffage, électricité, eau) & 26,5 & 8,7 & 13,6 & 6,3 \\
\hline C5 & Meubles et appareils ménagers & 6,1 & 9,2 & 7,8 & 1,2 \\
\hline C6 & Dépenses de santé & 5,7 & 3,9 & 5,0 & 0,9 \\
\hline C7 & Transports & 6,7 & 19,9 & 16,5 & 4,4 \\
\hline C8 & Communication & 3,3 & 2,6 & 2,8 & 0,3 \\
\hline C9 & Loisirs et culture & 9,7 & 14,0 & 13,1 & 1,7 \\
\hline C10 & Enseignement & 0,0 & 1,5 & 0,9 & 0,6 \\
\hline C11 & Restaurants et hôtels & 5,3 & 5,7 & 5,9 & 0,6 \\
\hline C12 & Autres (assurances, soins corporels, ...) & 10,4 & 12,1 & 10,2 & 0,7 \\
\hline & 2010 & Décile 1 & Décile 10 & Royaume & Ecart-type \\
\hline C1 & Alimentation et boissons non alcoolisées & 16,7 & 14,4 & 15,3 & 1,0 \\
\hline C2 & Alcool et tabac & 2,9 & 2,1 & 2,4 & 0,5 \\
\hline C3 & Articles d'habillement et chaussures & 3,3 & 6,5 & 5,3 & 1,3 \\
\hline C4 & Habitation (loyers, chauffage, électricité, eau) & 29,8 & 9,5 & 14,6 & 7,0 \\
\hline C5 & Meubles et appareils ménagers & 5,3 & 9,5 & 7,9 & 1,7 \\
\hline C6 & Dépenses de santé & 5,5 & 5,2 & 5,5 & 0,6 \\
\hline C7 & Iransports & 7,2 & 16,2 & 15,4 & 4,0 \\
\hline C8 & Communication & 3,6 & 2,5 & 3,0 & 0,5 \\
\hline C9 & Loisirs et culture & 9,1 & 15,0 & 12,6 & 1,9 \\
\hline C10 & Enseignement & 0,0 & 1,7 & 0,9 & 0,5 \\
\hline C11 & Restaurants et hôtels & 5,2 & 6,5 & 6,0 & 0,6 \\
\hline C12 & Autres (assurances, soins corporels, ...) & 11,3 & 10,1 & 11,3 & 1,0 \\
\hline & & & & \\
\hline
\end{tabular}

Les données du tableau 2 confirment notre idée de départ, à savoir que la composition du panier des biens et services achetés par les ménages à faible revenu diffère sensiblement de celle du panier acheté par les ménages à revenu élevé. Ainsi, les dépenses d'alimentation et d'habitation représentent une part plus importante de la dépense pour les ménages à faible revenu que pour les ménages à revenu élevé. La différence la plus forte concerne les dépenses d'habitation (C4), qui comprennent le loyer ainsi que les frais de chauffage, d'eau et d'électricité. En prenant les données de l'EBM de 2010, il apparaît en effet que pour les ménages du décile 1, ces dépenses absorbent environ $30 \%$ des dépenses totales du ménage, alors qu'elles 
... Méthodologie

Tableau 3. Structure des dépenses des ménages par catégorie d'âge

(en \%)

Source : SPF Economie et calcul des auteurs ne représentent que $10 \%$ des dépenses totales des ménages du décile 10. Pour ce qui concerne les dépenses dont la part est plus importante pour les ménages à haut revenu que pour les ménages à faible revenu, l'écart est le plus marqué pour les dépenses de loisirs et culture (C9) et pour les dépenses de transport (C7) : en 2010 , les ménages à faible revenu ont affecté $9,1 \%$ de leurs dépenses totales aux loisirs et 7,2\% aux dépenses de transport, alors que la part de ces dépenses fut de respectivement $15,0 \%$ et $16,2 \%$ pour les ménages à haut revenu.

Un autre résultat important que met en évidence le tableau 2 est la variation parfois importante dans le temps de certaines pondérations. Ainsi, entre 2000 et 2010, la partie des dépenses totales consacrées par les ménages du décile 1 au logement est passée de 26,5\% à 29,8\%, alors qu'elle a augmenté légèrement (de 8,7 \% à 9,5\%) pour les ménages du décile 10. La part des dépenses de transport a baissé pour les ménages du décile 10 , alors qu'elle a augmenté pour les ménages du décile 1 . La part des dépenses de santé a augmenté pour tous les déciles sauf le décile 1. En ce qui concerne les autres catégories de dépenses, leur part a généralement diminué pour la plupart des ménages sur la période considérée.

Le tableau 3 présente la structure des dépenses de biens et services, en 2000 et en 2010, pour les ménages de 25-29 ans et pour les ménages de 70 ans et plus, ainsi

\begin{tabular}{|c|c|c|c|c|c|}
\hline & 2000 & $25-29$ & 70 et + & Royaume & Ecart-ype \\
\hline $\mathrm{Cl}$ & Alimentation et boissons non alcoolisées & 12,4 & 18,3 & 15,6 & 2,1 \\
\hline C2 & Alcool et tabac & 2,3 & 3,2 & 2,9 & 0,6 \\
\hline C3 & Arricles d'habillement et chaussures & 5,3 & 4,5 & 5,8 & 1,0 \\
\hline C4 & Habitation (loyers, chauffage, êlectricité, eau) & 18,7 & 20,2 & 13,6 & 5,0 \\
\hline$c 5$ & Meubles et appareils ménagers & 6,3 & 7,6 & 7,8 & 1,2 \\
\hline C6 & Dépenses de santé & 3,2 & 8,8 & 5,0 & 2,0 \\
\hline C7 & Transports & 20,1 & 9,5 & 16,5 & 5,2 \\
\hline C8 & Communication & 3,0 & 2,6 & 2,8 & 1,0 \\
\hline c9 & Loisiss et culture & 12,2 & 9,4 & 13,1 & 1,9 \\
\hline $\mathrm{C} 10$ & Enseignement & 0,0 & 0,0 & 0,9 & 0,8 \\
\hline $\mathrm{C} 11$ & Restaurants et hôtels & 5,9 & 5,7 & 5,9 & 1,5 \\
\hline \multirow[t]{2}{*}{$\mathrm{Cl2}$} & Autres (assurances, soins corporels, ...) & 10,6 & 10,2 & 10,2 & 2,3 \\
\hline & 2010 & $25-29$ & 70 et + & Royaume & Ecorrt-type \\
\hline $\mathrm{Cl}$ & Alimentation et boissons non alcoolisées & 12,6 & 18,3 & 15,3 & 1,8 \\
\hline C2 & Alcool et tabac & 2,2 & 2,9 & 2,4 & 0,6 \\
\hline C3 & Articles d'habillement et chaussures & 5,5 & 3,6 & 5,3 & 1,1 \\
\hline C4 & Habitation (loyers, chauffage, électricitié, eau) & 19,6 & 18,4 & 14,6 & 6,1 \\
\hline 15 & Meubles et appareils ménagers & 5,4 & 8,7 & 7,9 & 1,8 \\
\hline C6 & Dépenses de santé & 3,9 & 8,7 & 5,5 & 1,7 \\
\hline C7 & Transports & 15,6 & 7,5 & 15,4 & 4,3 \\
\hline C8 & Communication & 3,5 & 2,6 & 3,0 & 0,3 \\
\hline C9 & Loisirs et culture & 11,5 & 11,0 & 12,6 & 1,8 \\
\hline $\mathrm{C} 10$ & Enseignement & 0,0 & 0,0 & 0,9 & 0,8 \\
\hline $\mathrm{C} 11$ & Restaurants et hôtels & 7,3 & 6,7 & 6,0 & 0,6 \\
\hline $\mathrm{C} 12$ & Autres (assurances, soins corporels, ...) & 12,9 & 11,3 & 11,3 & 2,2 \\
\hline
\end{tabular}


... Méthodologie

2. Inflation selon le revenu que pour l'ensemble des ménages du Royaume. On constate des différences significatives dans la structure de consommation selon les groupes d'âge. En 2010, les ménages de 25-29 ans ont affecté 15,6\% de leurs dépenses totales aux transports (C7) contre $7,5 \%$ pour le groupe d'âge 70 ans et plus. A l'inverse, en 2010 , les ménages de $\geq 70$ ans ont consacré une plus grande part de leurs dépenses à l'alimentation (C1) que les ménages de 25-29 ans : 18,3\% contre 12,6\%. La part des dépenses de santé est presque trois fois plus grande chez les $\geq 70$ ans que chez les 25-29 ans. Comme pour le tableau 2, on observe aussi des variations parfois importantes dans le temps de la structure de dépense pour chaque groupe d'âge.

Le tableau 4 présente le taux d'inflation générale calculé pour chacun des 10 déciles de ménages, et le graphique 1, ce même taux d'inflation cumulé entre 2001 et 2011. Dans la mesure où les mécanismes d'indexation qui sont en vigueur en Belgique prennent en compte l'évolution de l'indice «santé», plutôt que celle de l'indice général des prix à la consommation, le graphique 1 reprend également l'inflation cumulée mesurée en utilisant l'indice «santé». Il s'agit en fait du taux d'inflation calculé pour l'ensemble des ménages en ne tenant pas compte de l'évolution des prix du tabac, de l'alcool et des carburants ${ }^{18}$. C'est en Belgique le taux d'inflation qui, depuis 1994, sert de référence pour le calcul de l'indexation d'un grand nombre de travailleurs (cf. infra). Comme ce taux est identique pour chaque groupe de revenu, il permet de mesurer l'écart entre l'impact de l'indexation sur le pouvoir d'achat et l'inflation effectivement supportée pour chaque catégorie de revenu.

Les résultats obtenus sont assez différents de ceux présentés dans notre étude antérieure. Pour rappel, dans notre étude de 2006, nous avions constaté des écarts d'inflation assez faibles selon le revenu. Par exemple, le taux d'inflation globale observé pour les ménages du décile 1 entre 1998 et 2005 était de 14,6\%, tandis que celui observé pour les ménages du décile 10 était de $13,8 \%$. Ce résultat n'est manifestement plus valable aujourd'hui puisque, comme l'indique clairement le graphique 2 ci-dessous, les écarts d'inflation se sont creusés depuis 2004, pour aboutir à un taux cumulé d'inflation sur la période $2001-2011$, de $32 \%$ pour les ménages pauvres du décile 1 contre $26 \%$ pour les ménages riches du décile 10 . Pour toutes les autres catégories de revenu, l'inflation cumulée est décroissante avec le niveau de revenu.

Une autre observation intéressante est que l'inflation effective dépasse, pour chaque décile de ménage, le taux d'inflation qui détermine l'indexation. Ceci suggère une indexation insuffisante pour maintenir le pouvoir d'achat inchangé. L'écart est le plus grand pour les ménages du décile 1 avec une inflation globale de $32 \%$ et une indexation mesurée par l'indice santé de $24 \%$ sur la même période. Ce qui représente une perte de pouvoir d'achat potentielle entre 2001-2011 de $8 \%$. L'écart est néanmoins le plus faible pour les ménages du décile 10 avec une inflation globale de $26 \%$ et une indexation de $24 \%$. Pour les ménages du décile 10 , la perte de pouvoir d'achat sur la période est de seulement $2 \%$.

Une précision s'impose concernant l'indexation. Il convient en effet de distinguer d'un côté les fonctionnaires et les allocations sociales, et de l'autre côté les salariés du privé. C'est l'indice-pivot qui déclenche les augmentations des salaires dans la fonction publique et des allocations sociales. Dès lors que l'indice-pivot est dépassé, les traitements des fonctionnaires, les pensions et les allocations (chômage, revenu d'intégration, etc.) augmentent de $2 \%$. Dans le secteur privé, ce n'est pas

\footnotetext{
${ }^{18}$ Dans notre définition de l'indice «santé», tous les carburants sont exclus, alors que dans l'indice «santé» officiel, le LPG n'est pas retiré.
} 
... Inflation selon le revenu aussi simple. Il n'existe pas de cadre légal définissant les mécanismes d'indexation des salaires qui fait l'objet d'un règlement sectoriel. Chaque secteur est libre de conclure une convention collective de travail au sein de sa commission paritaire et de fixer les modalités de son propre mécanisme d'indexation. Chaque employeur est ensuite tenu d'appliquer ce mécanisme d'indexation spécifique. ${ }^{19}$ Il convient toutefois de préciser que les modalités particulières des différents mécanismes d'indexation n'ont que très peu d'impact sur l'indexation cumulée de long-terme comme étudiée dans notre analyse.
Tableau 4. Taux d'inflation générale cumulée par catégorie de revenu (en \%)

Source : SPF Economie et calcul des auteurs

\begin{tabular}{cccccccccccc}
\hline & Décile 1 & Décile 2 & Décile 3 & Décile 4 & Décile 5 & Décile 6 & Décile 7 & Décile 8 & Décile 9 & Décile 10 \\
\hline 2001 & 2,2 & 2,7 & 2,5 & 2,8 & 2,9 & 2,3 & 2,4 & 2,4 & 2,4 & 2,6 \\
\hline 2002 & 3,6 & 3,7 & 3,7 & 3,9 & 4,1 & 3,7 & 3,8 & 3,7 & 3,8 & 3,9 \\
\hline 2003 & 5,3 & 5,5 & 5,4 & 5,5 & 5,5 & 5,0 & 5,2 & 5,1 & 4,9 & 5,3 \\
\hline 2004 & 7,6 & 7,7 & 7,3 & 7,6 & 7,6 & 7,0 & 7,1 & 6,9 & 6,8 & 7,0 \\
\hline 2005 & 10,4 & 10,4 & 10,1 & 10,4 & 10,4 & 9,5 & 9,6 & 9,3 & 9,3 & 9,4 \\
\hline 2006 & 13,7 & 14,2 & 13,3 & 13,2 & 13,0 & 12,5 & 12,6 & 11,7 & 11,8 & 11,9 \\
\hline 2007 & 16,8 & 17,5 & 16,0 & 16,0 & 15,0 & 14,4 & 14,7 & 14,3 & 14,0 & 14,1 \\
\hline 2008 & 22,4 & 22,0 & 21,1 & 21,2 & 20,0 & 19,7 & 19,2 & 18,4 & 18,2 & 18,0 \\
\hline 2009 & 22,4 & 21,9 & 21,1 & 20,6 & 20,9 & 19,9 & 19,9 & 18,4 & 18,9 & 18,1 \\
\hline 2010 & 25,0 & 24,3 & 23,8 & 23,7 & 23,5 & 22,7 & 22,1 & 22,0 & 21,7 & 21,2 \\
\hline 2011 & 31,8 & 30,9 & 28,9 & 28,8 & 28,5 & 27,3 & 26,9 & 26,8 & 25,8 & 25,6 \\
\hline
\end{tabular}

Graphique 1. Taux d'inflation générale cumulée par catégorie de revenu et taux d'inflation cumulée de l'indice santé (en \%), 2001-2011

Source : SPF Economie et calcul des auteurs

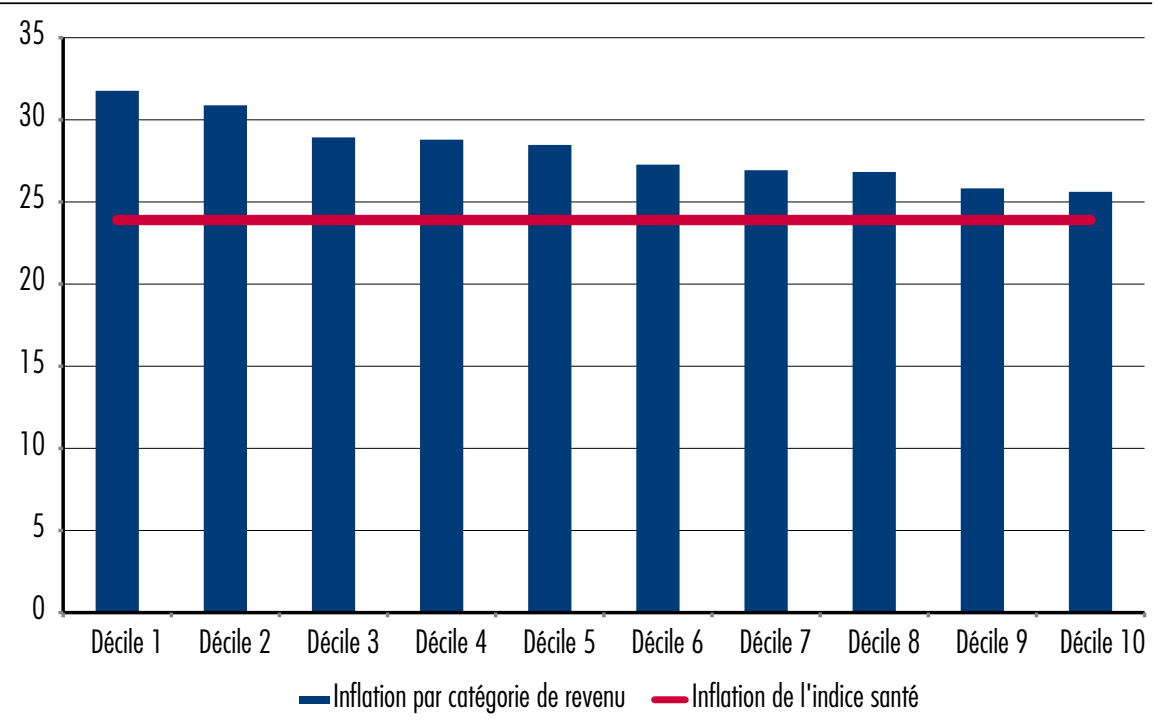

\footnotetext{
${ }^{19}$ Il existe deux grands mécanismes d'indexation dans le secteur privé : l'indexation à intervalles fixes, selon un pourcentage variable, et l'indexation à intervalles variables, sur base d'un pourcentage fixe, l'indice-pivot, comme c'est le cas pour l'indexation des salaires des fonctionnaires et des allocations sociales. Dans le cas de l'indexation à un moment fixe, la fréquence de l'indexation varie d'un secteur à l'autre : dans certains d'entre eux, les salaires ne sont indexés qu'une fois par an, tandis que d'autres ont opté pour une indexation trimestrielle, bimensuelle ou mensuelle. En clair, il revient à chaque commission paritaire de fixer le moment où les salaires seront indexés. La seconde méthode d'indexation, qui recourt à l'indice-pivot, s'applique à un peu plus de $40 \%$ des travailleurs du secteur privé.
} 


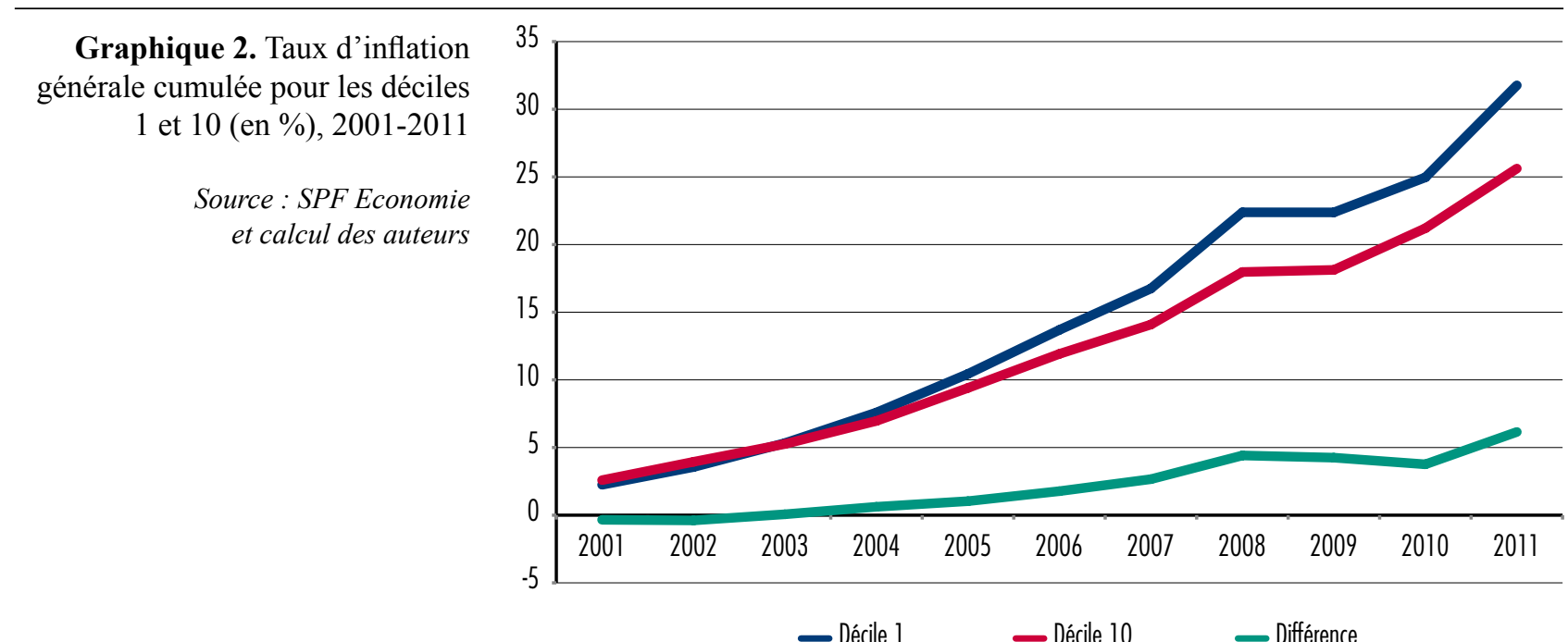

Afin de comprendre pourquoi l'inflation supportée par les ménages à faible revenu diffère autant de l'inflation supportée par les ménages à haut revenu, nous avons calculé, pour chaque groupe de produits, sa contribution à la mesure du taux d'inflation générale.$^{20}$ Les contributions obtenues pour les ménages du décile 1 et du décile 10 sont présentées dans le tableau 5 pour la période 2001-2011. La première colonne indique la contribution en valeur de chaque groupe de produits à l'inflation globale pour les ménages du décile 1 et pour les ménages du décile 10. La seconde colonne indique les contributions en pourcentage de chaque groupe de produits à l'inflation globale pour les ménages du décile 1 et du décile 10 .

Les principales observations que l'on peut tirer du tableau 5 sont les suivantes :

- Parmi les 12 groupes de produits retenus, ceux qui contribuent le plus au taux d'inflation générale, quel que soit le décile de ménage considéré, sont le groupe des Produits alimentaires et boissons (C1), le groupe des Dépenses d'habitation (C4), le groupe des Dépenses liées au Transport (C7) et le groupe composite «Autres dépenses» (C12).

- Les biens du groupe $\mathrm{C} 4$ (Dépenses d'habitation) contribuent davantage à l'inflation des ménages du décile 1 qu'à celle des ménages du décile 10. En fait ce groupe de produits explique à lui seul deux tiers de l'inflation des ménages du décile 1 , contre à peine un cinquième de l'inflation des ménages du décile 10. Comme indiqué dans le tableau 1 , les biens du groupe $\mathrm{C} 4$ sont aussi les biens dont le prix a le plus augmenté avec une inflation cumulée de $53 \%$ sur la période 2001-2011. Le surcroît d'inflation pour les ménages du décile 1 trouve donc une bonne partie de son explication dans la forte hausse des prix liés aux dépenses d'habitation (voir section 4 pour plus de détails).

- Pour les autres catégories de biens, leur contribution à l'inflation générale est habituellement plus faible pour les ménages du décile 1 que pour les ménages du décile 10 , ce qui a pour effet de réduire sensiblement l'écart d'inflation entre les ménages du décile 1 et ceux du décile 10 sans pour autant éliminer cet écart qui reste important. Le cas où la différence est la plus forte est celui des dépenses d'alimentation (C1) : sur la période 2001-2011, leur contribution à l'inflation fut de 5,3 points de pourcentage pour les ménages du décile 10 contre 2,7 points pour les ménages du décile 1 (voir tableau 5). Un autre poste où la différence est forte entre les déciles est celui des dépenses de restaurants et hôtels (C11) qui contribuent à

${ }^{20}$ La contribution d'une catégorie des biens donnée à l'inflation cumulée est calculée selon la méthodologie présentée dans l'encadré 1. 


\section{... Inflation selon le revenu}

l'inflation globale pour 3,4 points de pourcentage pour les ménages du décile 10 contre 1,3 point de pourcentage pour les ménages du décile 1 . Les dépenses de transports $(\mathrm{C} 7)$ ont une contribution comparable à l'inflation pour les ménages du décile 1 et du décile 10 . Ces dépenses représentent autour de $12 \%$ de l'inflation globale de chaque catégorie de ménage.

En résumé, il apparaît que les dépenses d'habitation ont pour effet de relever l'inflation des ménages à faible revenu par rapport aux ménages à haut revenu, mais que cet effet est en partie compensé par les autres catégories de dépenses, en particulier les dépenses d'alimentations (C1), les dépenses de restaurant (C11) et les autres dépenses (C12).
Tableau 5. Contribution des différentes catégories de dépenses à l'inflation selon le revenu

Source : SPF Economie et calcul des auteurs

\section{Inflation selon l'âge}

\begin{tabular}{lllcccc}
\hline & & \multicolumn{2}{c}{ Décile l } & \multicolumn{2}{c}{ Décile 10 } \\
\hline C1 & Alimentation et boissons non alcoolisées & 2,7 & 8,5 & 5,3 & 20,8 \\
\hline C2 & Alcool et tabac & 0,3 & 0,8 & 0,4 & 1,7 \\
\hline C3 & Articles d'habillement et chaussures & $-1,0$ & $-3,3$ & $-0,4$ & $-1,4$ \\
\hline C4 & Habitation (loyers, chauffage, électricité, & 21,6 & 68,0 & 5,0 & 19,4 \\
\hline C5 & Meub) & 1,8 & 5,6 & 1,5 & 6,0 \\
\hline C6 & Dépenses de santé & 0,2 & 0,5 & 1,4 & 5,6 \\
\hline C7 & Transports & 3,8 & 12,1 & 2,8 & 11,0 \\
\hline C8 & Communnication & $-0,1$ & $-0,4$ & $-0,3$ & $-1,3$ \\
\hline C9 & Loisirs et culture & $-1,5$ & $-4,7$ & 2,0 & 7,7 \\
\hline C10 & Enseignement & 0,0 & 0,0 & 0,9 & 3,4 \\
\hline C11 & Restaurants et hôtels & 1,3 & 4,0 & 3,4 & 13,2 \\
\hline C12 & Autres (assurances, soins corporels, ...) & 2,8 & 8,9 & 3,5 & 13,8 \\
\hline & Taux d'inflation générale & 31,8 & 100,0 & 25,6 & 100,0 \\
\hline
\end{tabular}

Le tableau 6 présente le taux d'inflation générale calculé pour chaque catégorie d'âge entre -25 ans et $\geq 70$ ans. Le graphique 3 présente ces mêmes taux cumulés sur la période 2001-2011 ainsi que l'inflation cumulée mesurée par l'indice «santé» et commune à toutes les catégories d'âge.

Les résultats obtenus sont les suivants. Le taux inflation est plus élevé pour les catégories d'âge extrêmes (-25 ans et $\geq 70$ ans) que pour les catégories d'âges intermédiaires. En fait, l'inflation semble d'abord diminuer à partir de 25 ans pour rester ensuite relativement stable jusque 50 ans et ensuite remonter jusqu'au niveau observé avant 25 ans. Les ménages à la pension (+65 ans) subissent l'inflation la plus forte. Le taux d'inflation supporté entre 2001-2011 par les ménages au-delà de 65 ans est proche de $32 \%$ contre un taux proche de $25 \%$ entre 25 et 34 ans. L'écart d'inflation entre ces deux catégories d'âges en 2011 est de 7 \% (soit un écart comparable à celui observé entre le décile 1 et le décile 10 des revenus).

Il ressort du graphique 3 que l'inflation effective dépasse, pour chaque catégorie d'âge, le taux d'inflation de l'indice «santé», qui détermine l'indexation. Ceci suggère une indexation insuffisante par rapport à l'inflation effective subie pour chaque catégorie d'âge. L'écart est le plus grand pour les ménages de +65 ans avec 
... Inflation selon l'âge une inflation globale de $32 \%$ et une indexation sur la période de $24 \%$. Ce qui représente une perte de pouvoir d'achat potentielle sur la période 2001-2011 de $8 \%$. L'écart est le plus faible pour les ménages entre 25-35 ans avec une inflation globale de $25 \%$ et une indexation de $24 \%$. Pour ces ménages, la perte potentielle de pouvoir d'achat sur la période est donc limitée à $1 \%$.

Une dernière observation tirée du graphique 4 est que les inégalités d'inflation selon l'âge se sont creusées sur la deuxième moitié de la période (à partir de 2004) et avant la crise de 2008 .
Tableau 6. Taux d'inflation générale cumulée par catégorie d'âge (en \%)

Source : SPF Economie et calcul des auteurs

Graphique 3. Taux d'inflation générale cumulée par catégorie d'âge et taux d'inflation cumulée de l'indice santé (en \%), 2001-2011

Source : SPF Economie et calcul des auteurs

\begin{tabular}{ccccccccccccc}
\hline & $<25$ & $25-29$ & $30-34$ & $35-39$ & $40-44$ & $45-49$ & $50-54$ & $55-59$ & $60-64$ & $65-69$ & $\geq 70$ \\
\hline 2001 & 3,2 & 2,2 & 2,2 & 2,7 & 2,4 & 2,5 & 2,8 & 2,3 & 2,8 & 2,9 & 2,8 \\
\hline 2002 & 4,0 & 3,4 & 3,5 & 3,9 & 3,8 & 3,8 & 4,0 & 3,9 & 3,7 & 4,4 & 4,1 \\
\hline 2003 & 5,8 & 4,9 & 5,0 & 5,3 & 5,0 & 4,9 & 5,1 & 5,5 & 5,6 & 5,8 & 6,1 \\
\hline 2004 & 7,7 & 6,9 & 7,0 & 7,1 & 6,9 & 6,7 & 6,9 & 7,4 & 7,5 & 8,2 & 8,2 \\
\hline 2005 & 10,2 & 9,4 & 9,4 & 9,9 & 9,4 & 9,1 & 9,4 & 10,1 & 10,2 & 10,9 & 11,3 \\
\hline 2006 & 12,4 & 11,7 & 12,0 & 12,2 & 11,9 & 11,4 & 12,8 & 13,3 & 13,7 & 14,4 & 14,5 \\
\hline 2007 & 15,7 & 13,5 & 14,5 & 14,3 & 13,8 & 13,8 & 14,6 & 16,0 & 15,7 & 16,9 & 18,0 \\
\hline 2008 & 21,3 & 17,5 & 18,5 & 17,7 & 18,7 & 19,2 & 19,5 & 19,8 & 21,4 & 22,6 & 23,0 \\
\hline 2009 & 21,8 & 18,8 & 18,2 & 19,0 & 18,6 & 19,2 & 19,4 & 21,2 & 20,5 & 21,7 & 22,9 \\
\hline 2010 & 24,7 & 21,0 & 20,8 & 21,4 & 21,9 & 22,0 & 22,0 & 23,3 & 24,1 & 23,8 & 26,4 \\
\hline 2011 & 28,1 & 25,3 & 25,9 & 26,0 & 26,4 & 26,4 & 26,1 & 30,0 & 28,7 & 31,5 & 32,1 \\
\hline
\end{tabular}

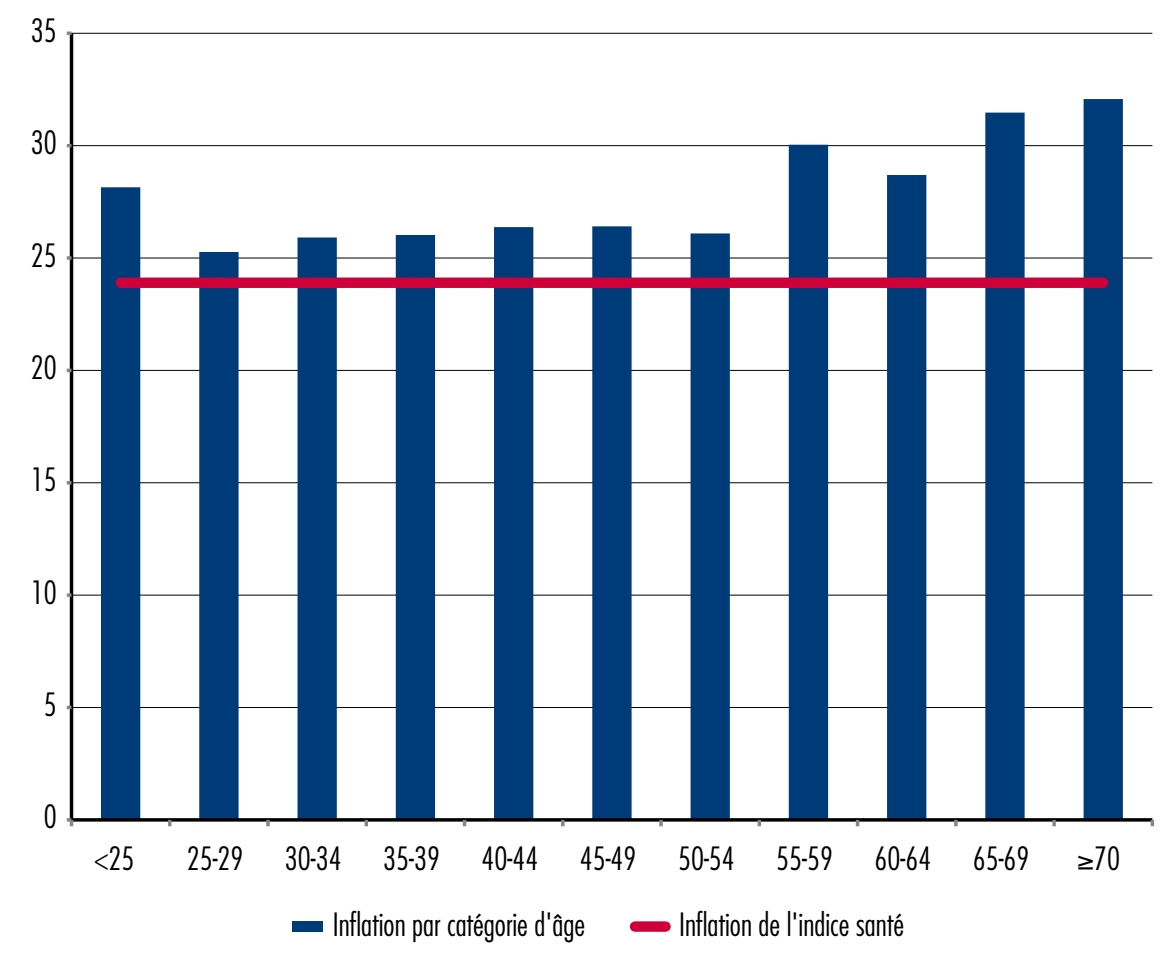


Graphique 4. Taux d'inflation générale cumulée pour les

25-29 ans et les $\geq 70$ ans (en \%), 2001-2011

Source : SPF Economie et calcul des auteurs

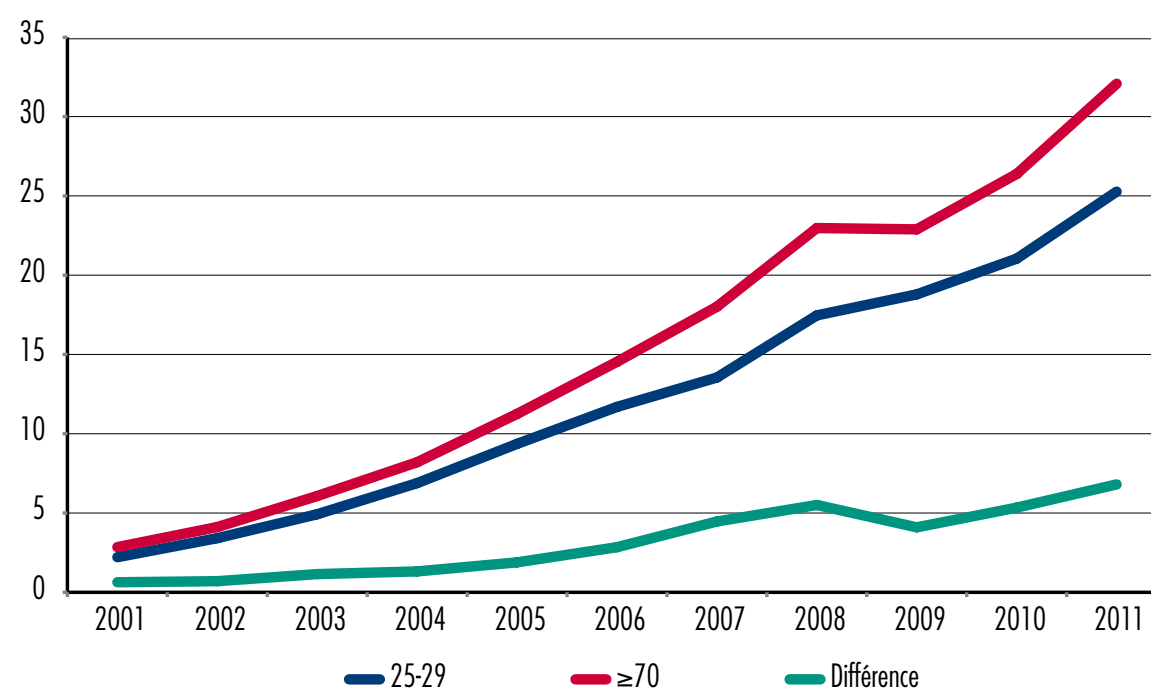

Afin de comprendre pourquoi l'inflation supportée par les ménages âgés diffère autant de l'inflation supportée par les ménages en début de carrière (25-29 ans), nous avons calculé, pour chaque groupe de produits, sa contribution à la mesure du taux d'inflation générale. Les contributions obtenues pour les ménages entre 25-29 ans et les ménages de $\geq 70$ ans sont présentées dans le tableau 7 (pour la période 2001-2011). La première colonne indique la contribution en valeur de chaque groupe de produits à l'inflation globale pour les ménages de chacune des deux catégories d'âge. La seconde colonne indique les contributions en pourcentage de chaque groupe de produits à l'inflation globale. Les principales observations que l'on peut tirer du tableau 7 sont les suivantes :

- Parmi les 12 groupes de produits retenus, ceux qui contribuent le plus au taux
Tableau 7. Contribution des différentes catégories de dépenses à l'inflation selon l'âge

Source : SPF Economie et calcul des auteurs

\begin{tabular}{llcccc}
\hline \multirow{2}{*}{} & & \multicolumn{2}{c}{$25-29$ ans } & \multicolumn{2}{c}{70 ans et + } \\
\cline { 3 - 6 } & & En valeur. & En p.c. & En valeur & En p.c. \\
\hline C1 & Alimentation et boissons non alcoolisées & 3,6 & 14,2 & 6,3 & 19,7 \\
\hline C2 & Alcool et tabac & 0,7 & 2,6 & 0,4 & 1,2 \\
\hline C3 & Articles d'habillement et chaussures & $-1,4$ & $-5,5$ & 0,2 & 0,6 \\
\hline C4 & $\begin{array}{l}\text { Habitation } \\
\text { (loyers, chauffage, électricité, eau) }\end{array}$ & 8,3 & 32,9 & 9,3 & 28,9 \\
\hline C5 & Meubles et appareils ménagers & 0,1 & 0,3 & 1,3 & 4,1 \\
\hline C6 & Dépenses de santé & 0,5 & 1,9 & 0,0 & 0,1 \\
\hline C7 & Transports & 4,9 & 19,2 & 5,2 & 16,3 \\
\hline C8 & Communication & $-0,1$ & $-0,4$ & $-0,3$ & $-1,0$ \\
\hline C9 & Loisirs et culture & 0,8 & 3,2 & 2,1 & 6,6 \\
\hline C10 & Enseignement & $-0,1$ & $-0,2$ & 0,0 & 0,0 \\
\hline C11 & Restaurants et hôtels & 3,2 & 12,5 & 2,8 & 8,8 \\
\hline C12 & Autres (assurances, soins corporels, ...) & 4,9 & 19,5 & 4,8 & 14,8 \\
\hline & Taux d'inflation générale & 25,3 & 100,0 & 32,1 & 100,0 \\
\hline
\end{tabular}


... Inflation selon l'âge

\section{L'influence des prix de l'énergie?}

d'inflation générale, quelle que soit la catégorie d'âge considérée, sont le groupe des Produits alimentaires et boissons (C1), le groupe des Dépenses d'habitation (C4), le groupe des Dépenses liées au Transport (C7), et le groupe composite «Autres dépenses» (C12). Ce constat est identique à celui établi dans la section précédente pour l'inflation selon le revenu.

- L'écart d'inflation entre les $\geq 70$ ans et le groupe des 25-29 ans est de 7 points de pourcent (respectivement $32 \%$ contre $25 \%$ ). On peut décomposer cet écart comme suit : 3 points de pourcent en dépenses d'alimentation $(\mathrm{C} 1)$, et 1 point de pourcent en dépenses sur chacun des postes suivants, habillement (C3), habitation (C4), mobilier et appareils (C5) et loisir et culture (C9). Assez curieusement, les dépenses de santé n'interviennent pas dans cet écart d'inflation selon l'âge, alors que ces dépenses représentent pourtant une part beaucoup plus importante de la consommation des ménages âgés que des ménages jeunes. La raison est simplement que l'inflation pour ce groupe de dépenses est restée assez faible sur la période, comme indiqué dans le tableau 1. Le surcroît d'inflation pour les ménages âgés trouve donc une bonne partie de son explication dans la forte hausse des prix liés aux dépenses d'alimentation.

Notre analyse a révélé que les écarts d'inflation entre les groupes extrêmes, à savoir d'une part entre les ménages les plus pauvres et les plus riches et, d'autre part, entre les ménages en début de carrière (25-29 ans) et les ménages pensionnés ( +65 ans), s'étaient creusés au cours de la période. Dans les deux cas, les inégalités d'inflation ont surtout augmenté entre 2004 et 2008, pour se stabiliser ensuite. Un fait macroéconomique marquant durant cette période, et dont on peut penser qu'il a eu un impact sur l'évolution des inégalités d'inflation, est la forte augmentation des prix de l'énergie. Entre 2004 et 2008, les prix de l'électricité ont en effet crû de $25 \%$, ceux du gaz de $60 \%$, ceux des combustibles liquides (comprenant le mazout de chauffage) d'un peu plus de $100 \%$.

Pour évaluer si ces hausses de prix ont eu pour effet de creuser les inégalités d'inflation, nous présentons, dans les graphiques 5 et 6 , la contribution des prix de l'énergie (et du logement) à l'inflation cumulée supportée par les ménages du décile 1 et du décile 10 d'une part, et par les ménages de 25-29 ans et les ménages de $\geq 70$ ans d'autre part. ${ }^{21}$ Il apparaît effectivement à l'analyse de ces graphiques que, entre 2004 et 2008 , la contribution des prix de l'énergie à l'inflation a augmenté nettement plus pour les ménages les plus pauvres et pour les ménages les plus âgés que pour, respectivement, les ménages les plus riches et les ménages âgés de 25 à 30 ans. Entre 2004 et 2008, l'écart d'inflation cumulée entre les ménages du décile 1 et du décile 10 a augmenté de 3,8 points de pourcentage (de $0,62 \%$ en 2004 à $4,41 \%$ en 2008) et les prix de l'énergie et du logement ont contribué à cette hausse à hauteur de 8,2 points de pourcentage (voir graphique 5) ${ }^{22}$. Sur la même période, l'écart d'inflation cumulée entre les ménages de $\geq 70$ ans et les ménages de 25-29 ans a augmenté de 4,2 points de pourcentage (de $1,32 \%$ en 2004 à $5,51 \%$ en 2008 ) et les prix de l'énergie et du logement ont contribué à cette hausse à hauteur de 5,3 points (voir graphique 6) ${ }^{23}$. Si l'on se réfère au tableau 7 (cf. section 3),

\footnotetext{
${ }^{21}$ Le coût des logements ayant augmenté de seulement $10 \%$ entre 2004 et 2008, son impact est marginal par rapport à celui des prix de l'énergie.

${ }^{22}$ Comme on peut le voir sur le graphique 5 , la contribution de l'énergie et du logement à l'inégalité d'inflation est de 4,8 points en 2004 et elle augmente à 13,0 points en 2008 . Cette contribution de 8,2 points de l'énergie et du logement à l'accroissement de l'inégalité d'inflation est compensée par une variation dans l'autre sens de la contribution d'autres postes de dépenses pour obtenir une variation finale de l'écart d'inflation de 3,8 points entre 2004 et 2008.

${ }^{23}$ La contribution des prix de l'énergie et du logement à l'inégalité d'inflation entre les 2 classes d'âge passe en effet de - 3,9 points en 2004 à 1,4 point en 2008 .
} 
... L'influence des prix de l'énergie?

les prix alimentaires sont ceux qui contribuent le plus aux inégalités d'inflation par âge lorsque l'on prend toute la période (2001-2011). En revanche, les prix de l'énergie et du logement sont ceux qui contribuent le plus à la variation de ces inégalités d'inflation entre 2004 et 2008.

Nos résultats tendent donc à montrer que les fortes hausses des prix de l'énergie sont un facteur important d'inégalité d'inflation entre ménages. En revanche, nos résultats indiquent que la crise économique de 2008-2009 n'a pas provoqué une augmentation des inégalités d'inflation. En fait, sur cette période, les inégalités d'inflation se sont légèrement réduites du fait d'une baisse des prix de l'énergie (le gaz, l'électricité et le mazout de chauffage ont baissé sur cette période). Malheureusement, les inégalités d'inflation sont reparties à la hausse depuis 2010 avec le retour à la hausse des prix de l'énergie.

Graphique 5. Contribution des prix de l'énergie et du logement

à l'indice général des prix des déciles 1 et $10(\mathrm{en} \%)$

Source : SPF Economie et calcul des auteurs

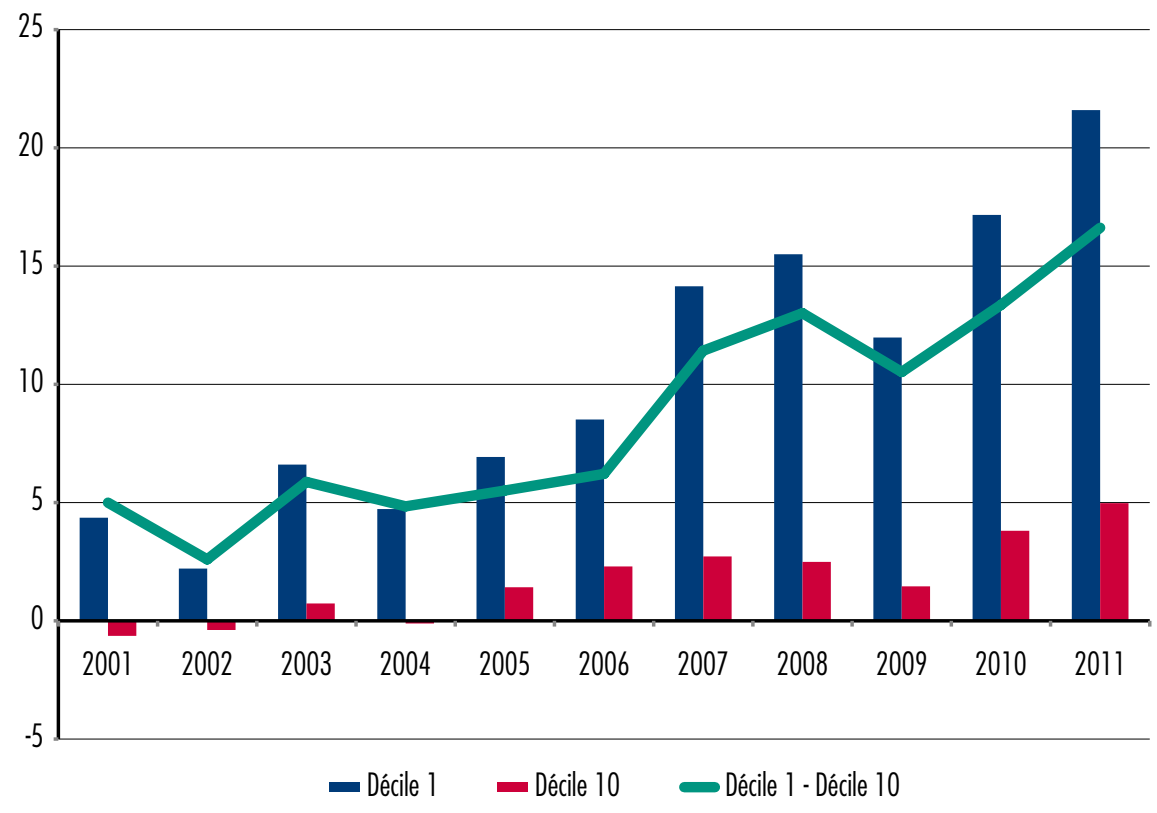

Graphique 6. Contribution des prix de l'énergie et du logement à

l'indice général des prix des 25-29 ans et des $\geq 70$ ans (en \%)

Source : SPF Economie et calcul des auteurs

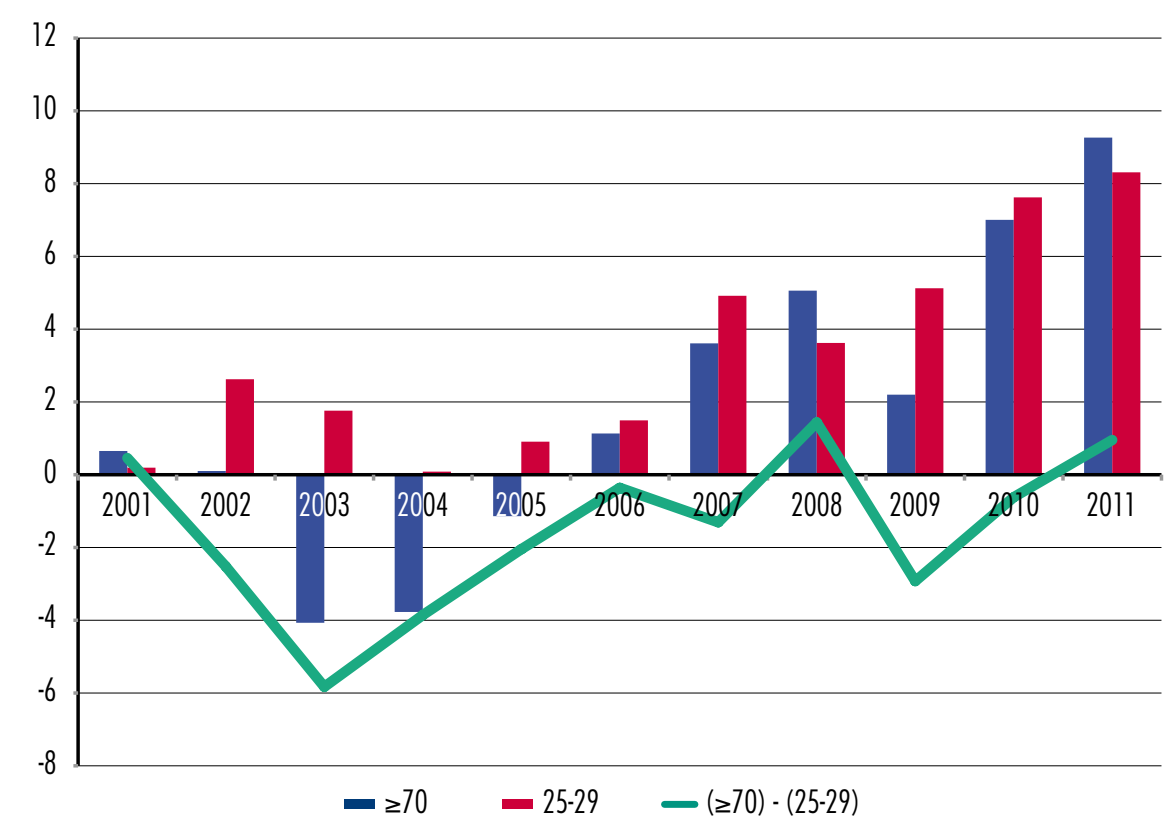




\section{Conclusion}

Vincent Bodart est professeur

d'économie à l'UCL et chercheur

à l'IRES (UCL).

Jean Hindriks est professeur

d'économie à l'UCL, senior fellow

à l'Itinera Institute et chercheur au

CORE (UCL).
Dans ce numéro de Regards économiques, nous avons essayé de calculer au plus juste l'inflation par groupe de ménages en distinguant les ménages selon leur revenu et selon leur âge. Pour calculer l'inflation au plus près, il ne s'agit pas seulement de suivre les prix et leurs évolutions, mais aussi les tendances en matière de consommation des différents groupes de ménage. Dans la mesure où les profils de consommation changent avec le revenu et avec l'âge, on doit s'attendre à ce que l'inflation change pour chaque catégorie de ménage. Nous avons réalisé cette étude pour la période 2001-2011 en regroupant les ménages par décile de revenu pour mesurer l'inflation selon le revenu. Nous avons aussi réalisé la même analyse en regroupant les ménages par catégorie d'âge pour mesurer l'inflation selon l'âge. Dans le premier cas, nous avons montré que l'inflation était décroissante avec le revenu. Les ménages du décile 1 ont une inflation cumulée proche de $32 \%$ contre une inflation cumulée de $26 \%$ pour les ménages du décile 10 . Dans le second cas, pour ce qui concerne l'âge, nous avons montré que l'inflation est d'abord décroissante avec l'âge pour ensuite remonter avec l'âge à partir de 55 ans. L'inflation supportée entre 2001 et 2011 par les ménages de 70 ans et plus atteint $32 \%$ contre une inflation de $25 \%$ pour les ménages entre 25-35 ans.

Nous avons ensuite analysé la contribution de chaque groupe de produits à ces écarts d'inflation entre les différentes catégories de ménage. Pour les écarts selon le revenu, les dépenses d'habitation sont la principale raison d'un surcroît d'inflation pour les bas revenus. Pour les écarts selon l'âge, les dépenses d'alimentation sont la principale source du surcroît d'inflation pour les ménages plus âgés (et non les dépenses de santé contrairement à ce que l'on aurait pu penser). Nous avons enfin comparé l'inflation des ménages à la croissance de l'indice santé qui est utilisé pour l'indexation des salaires, des traitements et des allocations sociales. Nous avons montré que sur la période 2001-2011, l'indexation (mesurée par l'indice santé) est de $24 \%$ ce qui signifie une perte de pouvoir d'achat potentielle de $9 \%$ en dix ans pour les ménages du décile 1 et les ménages de plus de 65 ans. Notre système d'indexation semble donc moins bien protéger les ménages les plus vulnérables. Nos résultats tendent aussi à montrer que les fortes hausses des prix de l'énergie entre 2004 et 2008, mais aussi plus récemment depuis 2010, ont contribué à creuser les inégalités d'inflation. Cette analyse suggère qu'il est extrêmement judicieux de mettre en place des systèmes tels que «les chèques mazout» pour protéger le pouvoir d'achat des plus vulnérables contre les hausses des prix de l'énergie. 
Références pour en savoir plus
Baldini Massimo (2005), «Inflation Inequality in Italy», article non publié.

Bodart Vincent et Jean Hindriks (2006), "Protéger le pouvoir d'achat à tout prix?", Regards économiques, 45, Novembre.

Bodart Vincent et Jean Hindriks (2006), "Les Belges sont-ils tous égaux face à l'inflation?", Regards économiques, 46, Novembre.

Crawford Ian et Zoë Smith (2002), «Distributional Aspects of Inflation», The Institute for Fiscal Studies, Commentary 90.

Hobjin Bart et David Lagakos (2003), «Inflation inequality in the United States», Federal Reserve Bank of New York Staff Reports, 173.

Izquierdo Mario, Eduardo Levy et Javier Ruiz-Castillo (2003), «The Plutocratic Gap in the CPI : Evidence from Spain», IMF Staff Papers, 50, 1.

Ministère des Affaires Economiques (1999), «L'indice des prix à la consommation, Base 1996», Administration de la politique commerciale, Service de l'indice.

Slesnick D.T. (1990), «Inflation, relative price variation, and inequality», Journal of Econometrics, 43, 135-151. 
Directeur de la publication :

Vincent Bodart

Rédactrice en chef :

Muriel Dejemeppe

Comité de rédaction : Paul Belleflamme,

Vincent Bodart, Thierry Bréchet, Muriel

Dejemeppe, Frédéric Docquier, Jean Hindriks,

Marthe Nyssens, William Parienté

Secrétariat \& logistique : Anne Davister

Graphiste : Dominos

\section{Regards Économiques IRES-UCL}

Place Montesquieu, 3

B1348 Louvain-la-Neuve

www.regards-economiques.be

regard-ires@uclouvain.be

tél. 010/4734 26

ISSN 2033-3013 